$07 ; 15$

\title{
Микроволновые усилители мощности на AIGaN/GaN-транзисторах \\ с двумерным электронным газом
}

(C) О.Г. Вендик ${ }^{1}$, И.Б. Вендик ${ }^{1}$, П.А. Туральчук ${ }^{1}$, Я.М. Парнес ${ }^{1}$, М.Д. Парнес ${ }^{2}$

${ }^{1}$ Санкт-Петербургский государственный электротехнический университет „ЛЭТИ“

${ }^{2}$ ООО „Резонанс“, Санкт-Петербург

E-mail: ogvendik@rambler.ru, mdparnes@mail.ru

Поступило в Редакцию 10 марта 2016 г.

Обсуждается методика синтеза микроволновых усилителей мощности на транзисторах с гетеропереходом AlGaN/GaN. Основное внимание уделено разработке методики синтеза трансформирующих цепей усилителя мощности с целью увеличения коэффициента полезного действия при сохранении высокого уровня выходной мощности. Используется независимое согласование на частотах гармоник и на фундаментальной частоте, что позволяет управлять значением достижимого коэффициента полезного действия в широкой полосе частот наряду с полным подавлением гармоник за пределами рабочей полосы. Разработаны и экспериментально исследованы микроволновые усилители мощности на 4 и $9 \mathrm{GHz}$.

С момента появления первых публикаций по разработке транзисторов на нитриде галлия GaN в компании APA Optics во главе с М. Ханом (M. Khan) [1] возможности приборов СВЧ-диапазона были существенно расширены благодаря использованию в составе транзисторной структуры гетероперехода $\mathrm{AlGaN} / \mathrm{GaN}$, обладающего уникальными свойствами. В зоне гетероперехода между барьерным 
$\mathrm{AlGaN}$-слоем и нелегированным $\mathrm{GaN}$-слоем формируется двумерный электронный газ с высокой подвижностью электронов [2]. Такой транзистор известен как транзистор с высокой подвижностью электронов (HEMT - High Electron Mobility Transistor). Высокая подвижность и высокая концентрация носителей заряда в канале $\mathrm{AlGaN} / \mathrm{GaN}$ наряду с широкой запрещенной зоной определяют частотные и мощностные свойства гетеропереходных полевых транзисторов. Применение $\mathrm{GaN}$-транзисторов существенно улучшает параметры СВЧ-усилителей мощности (УМ). УМ на HEMT-GaN-транзисторах используются в выходных каналах фазированных антенных решеток (ФАР), заменяя при этом гораздо более громоздкие и менее эффективные усилители мощности на клистронах или лампах бегущей волны [3]. ФАР это наиболее масштабные и дорогостоящие устройства современных радиоэлектронных систем. Отказ от вакуумных активных элементов в составе ФАР сильно упрощает их конструкцию. В настоящее время успешно выпускаются СВЧ-УМ на основе HEMT-GaN-транзисторов, выходная мощность которых достигает $1000 \mathrm{~W}$ [4].

Решающим фактором в развитии СВЧ-УМ является повышение коэффициента полезного действия (КПД) при сохранении линейности и расширении полосы частот устройства [5]. В последнее время научные исследования сосредоточены на ключевых режимах работы СВЧ-УМ, таких как классы Е и F, КПД которых достигает 70-80\% [6,7]. Основным физическим принципом увеличения КПД при работе усилителя в режиме ключа заключается в том, что в транзисторе, который переключается между состояниями открыт/закрыт, в каждый момент времени существует либо ноль напряжения, либо ноль тока. Режим работы УМ в классе J с высоким КПД, впервые предложенный в работе [8], отличается линейностью работы и расширенным диапазоном частот. В режиме класса Ј вторая гармоника используется в сочетании с импедансом фундаментальной частоты так, чтобы сформировать так называемый эффект „уплощения“ протекающего тока.

В данной работе основное внимание уделено разработке методики синтеза трансформирующих цепей УМ с целью достижения максимального КПД. В результате были разработаны и экспериментально исследованы микроволновые УМ мощностью $4.5 \mathrm{~W}$, предназначенные для работы на частотах 4 и $9 \mathrm{GHz}$ в непрерывном режиме. Полевой транзистор с затвором Шоттки (ГПТШ) с 10 ,пальцами“ и шириной

Письма в ЖТФ, 2016, том 42, вып. 21 


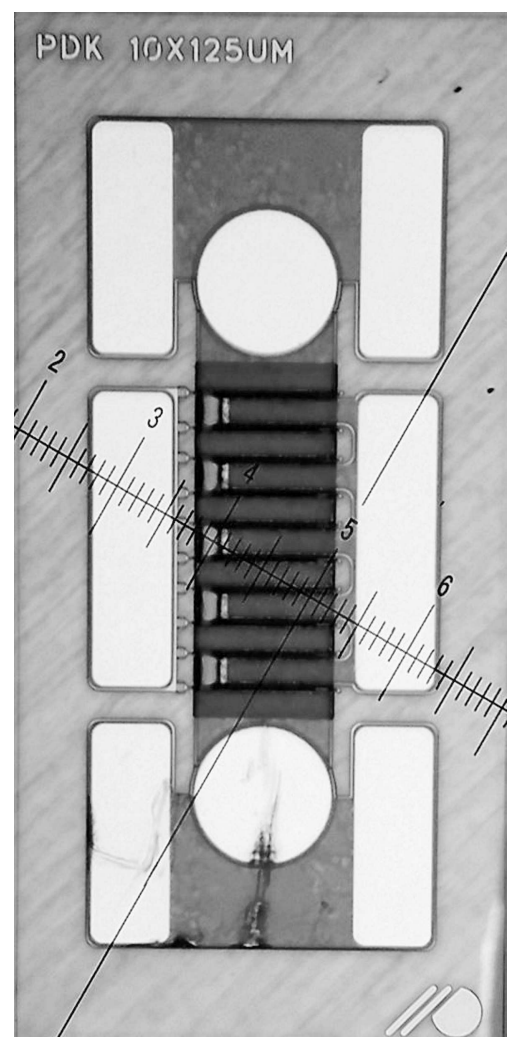

Рис. 1. Микрофотография AlGaN/GaN-HEMT-транзистора.

затвора $125 \mu$ m на основе гетероструктур $\mathrm{AlGaN} / \mathrm{GaN}$, использованный в составе УМ, приведен на рис. 1 .

Синтез СВЧ-усилителей в ключевых классах (режимы работы Е, F) с целью достижения максимально высокого КПД основан на анализе форм выходного тока и напряжения с целью минимизации их взаимного перекрытия [9]. Особый интерес представляет анализ работы УМ в частотной области с учетом конечного числа гармоник, обсуждаемый в работе [10]. Этот метод может быть применен к анализу и синтезу усилителей в режимах С, Е, F и J. При этом выполняется оценка

$1^{*}$ Письма в ЖТФ, 2016, том 42, вып. 21 

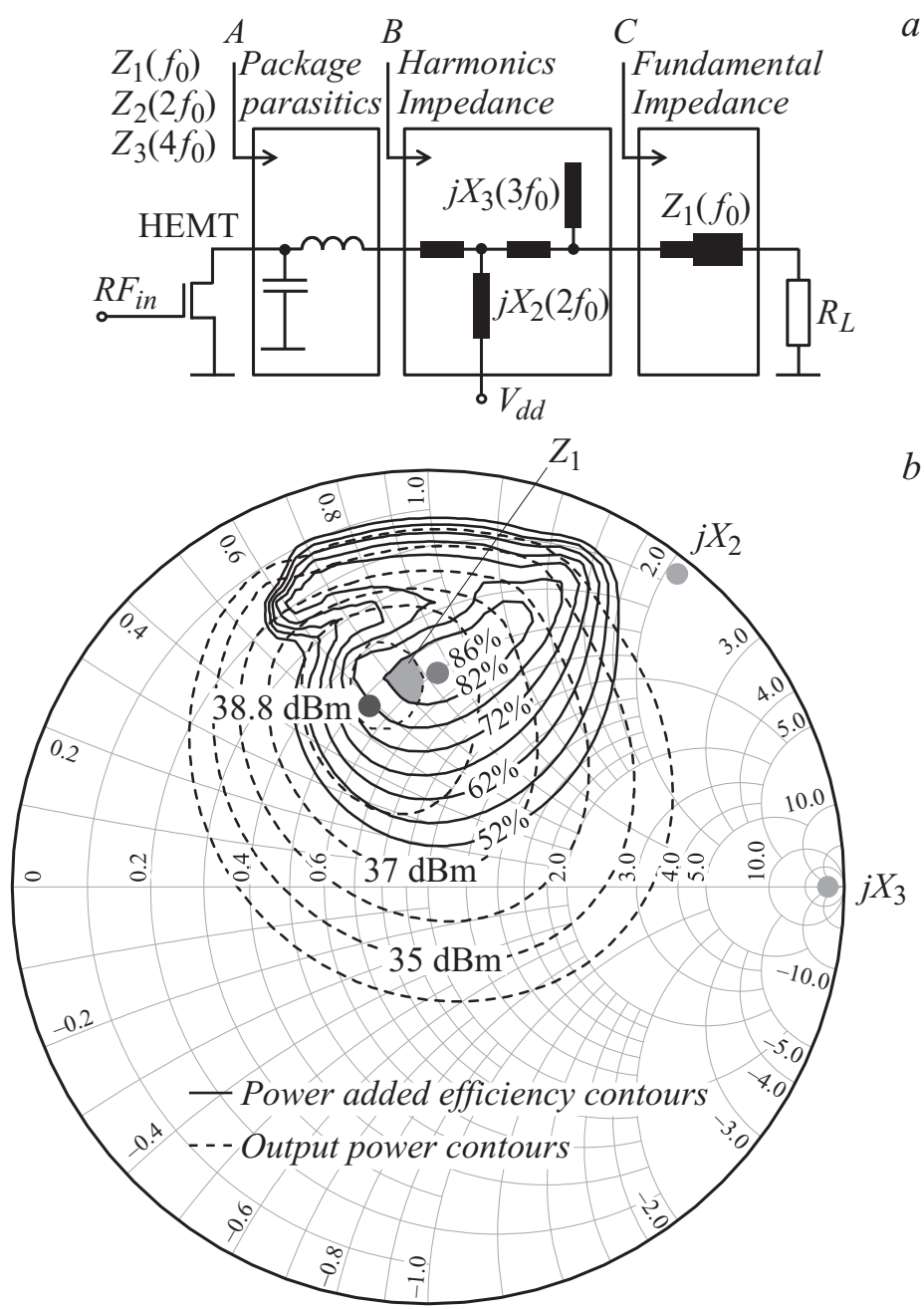

Рис. 2. Обобщенная схема УМ $(a)$. Результаты анализа импеданса нагрузки УМ на основной частоте и частотах гармоник, обеспечивающей максимальный КПД $(b)$. 
характеристик УМ, нагруженного на импеданс соответствующих гармоник. Обобщенная схема УМ, представленная транзистором и выходной согласующей цепью, приведена на рис. $2, a$. Количество гармонических составляющих, учитываемых в спектрах выходного сигнала, определяет максимально достижимый КПД. Для обеспечения заданного режима работы УМ с высоким КПД требуется анализ и расчет параметров выходной нагрузки транзистора с учетом гармонических составляющих. В то же время выходная мощность определяется комплексной нагрузкой на выходе усилителя на частоте основной гармоники. Расчет нагрузки СВЧ-УМ основан на использовании графоаналитического метода и оптимизационных процедур, заключающихся в построении контуров выходных нелинейных характеристик УМ, таких как выходная мощность и КПД (в англ. лит. load-pull technique) на традиционной диаграмме комплексных импедансов в полярных координатах. Анализ параметров СВЧ-транзистора с помощью данной методики позволяет определить оптимальную нагрузку транзистора для получения максимального КПД и выходной мощности. В случае работы транзистора в ключевом режиме, т.е. с использованием набора высших гармоник (классы Е, $\mathrm{F}, \mathrm{J})$, класс работы определяется параметрами согласующих цепей на частотах используемых гармоник. Это означает, что может быть найдено условие трансформации импеданса транзистора на основной частоте и реактивной нагрузки соответствующих гармоник. Контуры постоянной мощности и КПД, полученные на частоте основной гармоники, а также реактивные сопротивления на частотах второй и третьей гармоник $\left(j X_{2}\right.$ и $j X_{3}$ ) представлены на рис. 2, $b$. Пересечение контуров постоянной мощности и КПД позволяет определить границы области оптимальных нагрузок УМ на частоте основной гармоники.

Основной принцип синтеза согласующих цепей усилителя, принятый в данной работе, заключается в обеспечении соответствующей нагрузки транзистора как на частоте основной гармоники (плоскость $C$ ), так и с использованием набора высших гармоник (плоскость $B$, рис. 2, $a$ ) [11]. Данная методика независимого согласования на частотах гармоник и на фундаментальной частоте позволяет управлять значением достижимого КПД в широкой полосе частот наряду с полным подавлением гармоник за пределами рабочей полосы [12]. Благодаря существованию высокого значения реактивного сопротивления второй и третьей гармоник, параметры согласующей цепи гармоник в плоскости $B$ не зависят от

Письма в ЖТФ, 2016, том 42, вып. 21 

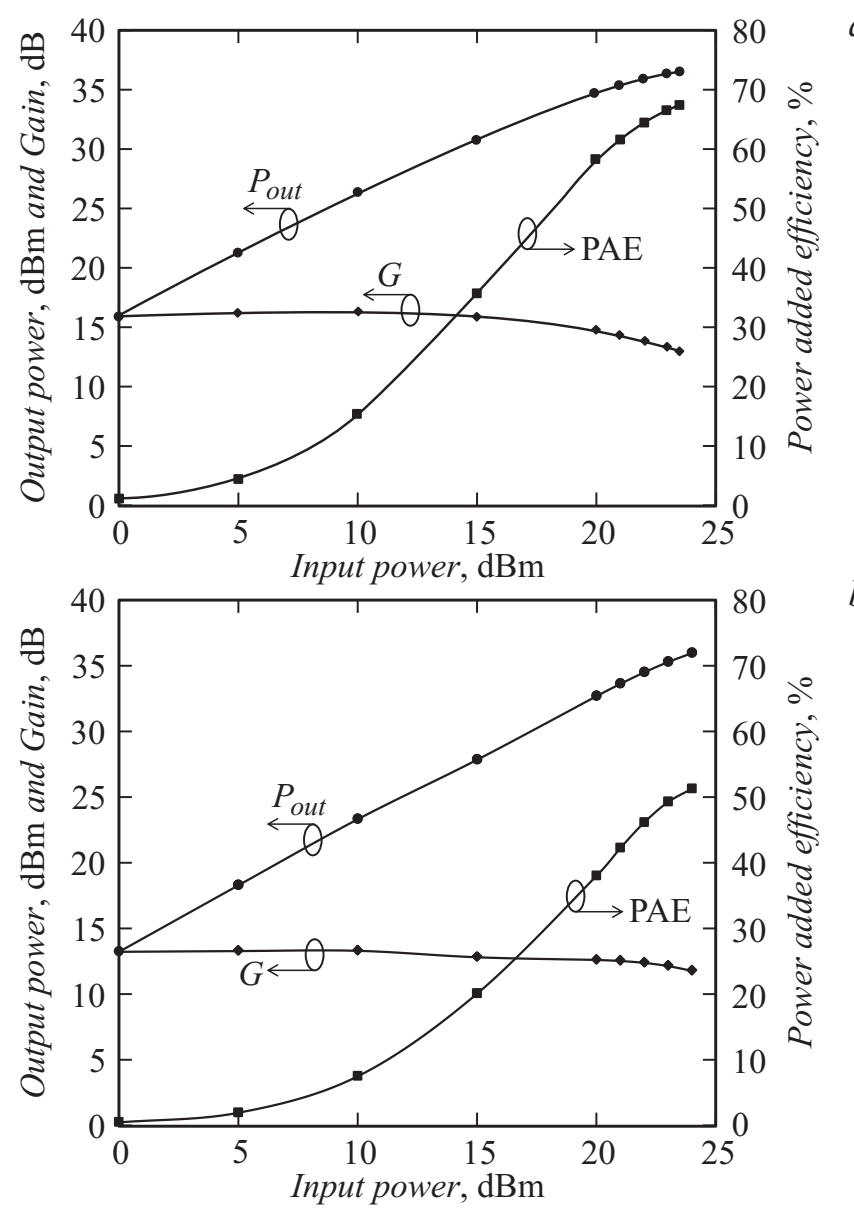

Рис. 3. Экспериментальные зависимости выходной мощности, коэффициента усиления и КПД от мощности входного сигнала УМ, разработанного для работы на $4 \mathrm{GHz}(a)$ и $9 \mathrm{GHz}(b)$.

параметров согласующих цепей на фундаментальной частоте в плоскости $C$ и полностью определяются параметрами транзистора на частотах гармоник. Выбор параметров согласующей цепи на фундаментальной 
частоте ведется с учетом компромисса между сохранением высокого значения КПД, максимальным подавлением гармоник в цепях нагрузки и поддержанием заданной выходной мощности.

В результате применения данной методики были разработаны однокаскадные усилители мощности СВЧ в виде интегральных схем, предназначенные для работы на частотах 4 и $9 \mathrm{GHz}$. Экспериментальное исследование УМ выполнено с использованием СВЧ-генератора Keysight E8663D и измерителя мощности Keysight U2044XA. Зависимости выходной мощности, коэффициента усиления и КПД УМ от мощности входного сигнала представлены на рис. 3. Усилитель на $4 \mathrm{GHz}$ характеризуется следующими параметрами: коэффициент усиления $12 \mathrm{~dB}$, КПД 68\%, выходная мощность $4.5 \mathrm{~W}$. УМ, предназначенный для работы на частоте $9 \mathrm{GHz}$, имеет выходную мощность $4 \mathrm{~W}$ и КПД не менее $45 \%$.

Таким образом, максимально достижимый КПД может быть получен с помощью расчета параметров усилителя мощности, нагруженного на импеданс соответствующих гармоник. Синтез трансформирующих цепей, обеспечивающих реактивную нагрузку на частотах второй и третьей гармоник и комплексный импеданс на фундаментальной гармонике, позволяет оптимизировать работу УМ с точки зрения уменьшения рассеиваемой мощности и соответственно повышения КПД.

Работа выполнена в рамках государственной работы „Проведение научно-исследовательских работ (фундаментальных научных исследований, прикладных научных исследований и экспериментальных разработок)“ проектной части государственного задания Министерства образования и науки РФ (задание № 8.2579.2014/К).

\section{Список литературы}

[1] Kuznia J.N., Khan M.A., Olson D.T., Kaplan R. // J. Appl. Phys. 1993. V. 73. N 27. P. $4700-4702$.

[2] Данилин В.Н., Жукова Т.А., Кузнещов Ю. и др. // Электроника. 2005. № 4. C. $20-29$.

[3] Борисов Л., Щелкунов Г. // Электроника. 2012. № 4. С. 102-110.

[4] Formicone G., Boueri F., Battaglia B. // Proc. IEEE COMCAS. 2013. P. 4.

[5] Grebennicov A. Switchmode RF and Microwave Power Amplifier. Academic Press, 2012. P. 345.

Письма в ЖТФ, 2016, том 42, вып. 21 
[6] Colantonio P., Giannini F., Limiti E. High efficiency RF and microwave solid state power amplifier. A John Wiley and Sons, 2009. P. 495.

[7] Pengelly R.S., Wood S.M., Milligan J.W. et al. // IEEE Trans. MTT. 2012. V. 60. N 6. P. $1764-1783$.

[8] Cripps S.C. RF Power Amplifiers for Wireless Communications. Artech House. Norwood, Mass, 2006.

[9] Sokal N.O. // IEEE MTT-S. 2000. V. 2. P. 779-782.

[10] Raab F.H. // IEEE Trans. MTT. 2001. V. 49. N 8. P. 1462-1468.

[11] Туральчук П.А., Кириллов В.В., Вендик О.Г., Парнес М.Д. // Труды конф. „Электроника и микроэлектроника СВЧ“. Санкт-Петербург, 2016. С. 182186.

[12] Tuffy N., Zhu A., Brazil T.J. // IEEE MTT-S. 2011. P. 1-4. 